\title{
Mild encephalitis/encephalopathy with a reversible splenial lesion in children
}

\section{Adalet Elçin Yıldız (D) Hülya Maraş Genç (i) Esra Gürkaş (1) Havva Akmaz Ünlü (D) İbrahim Halil Öncel (1) Alev Güven (D)}

From the Departments of Radiology (A.E.Y. $\triangle$ aelcindr@gmail.com, H.A.Ü.) and Pediatric Neurology (H.M.G., E.G., I.H.Ö., A.G.) University of Health Sciences, Ankara Child Health and Diseases Hematology Oncology Training and Research Hospital, Turkey.

Received 6 August 2017; revision requested 12 October 2017; last revision received 10 January 2018; accepted 16 January 2018.

DOI 10.5152/dir.2018.17319

\begin{abstract}
PURPOSE
We aimed to present clinical and radiologic characteristics of mild encephalitis/encephalopathy with a reversible splenial lesion (MERS) in children.
\end{abstract}

\section{METHODS}

Eight children ( 5 boys and 3 girls; median age, 5.9 years; age range, 8 months to 14.1 years) diagnosed with MERS between September 2015 and June 2017 were included in the study. We reviewed the patient's data, including demographic characteristics, prodromal and neurologic symptoms, neurologic examination, magnetic resonance imaging and electroencephalography findings, laboratory findings, treatment, and prognosis.

\section{RESULTS}

Prodromal symptoms were nausea and vomiting $(n=6)$, diarrhea $(n=6)$, and fever $(n=3)$. Initial neurologic symptoms were seizures $(n=4)$, delirious behavior $(n=1)$, drowsiness $(n=1)$, ataxia $(n=1)$, transient blindness $(n=2)$, abnormal speech $(n=2)$, and headache $(n=1)$. Two patients had a suspected infective agent: urinary tract infection caused by Escherichia coli and gastroenteritis caused by rotavirus. Seven patients had type I lesions, comprising characteristic symmetric ovoid $(n=6)$ and band-shaped $(n=1)$ T2-weighted hyperintense lesions at the spenium of corpus callosum, and one patient had type II lesion with additional symmetric posterior periventricular lesions. The lesions were isointense to mildly hypointense on T1-weighted imaging and did not show enhancement. All lesions displayed restricted diffusion. In all patients, neurologic symptoms completely normalized $<48$ hours from the onset of symptoms without any sequelae.

\section{CONCLUSION}

MERS has characteristic imaging features and favorable outcome.
$\mathrm{M}$ ild encephalitis/encephalopathy with a reversible splenial lesion (MERS) is a clinicoradiologic syndrome first described by Tada et al (1). Patients typically present with mild encephalopathy following prodromal symptoms such as fever, cough, vomiting and/or diarrhea. The most pronounced neurologic symptoms are disturbance of consciousness, abnormal speech, delirious behavior, seizures, muscle weakness, ophthalmoplegia, facial nerve paralysis, and headache $(2,3)$.

On magnetic resonance imaging (MRI), MERS is almost always associated with a transient splenial lesion that is slightly hyperintense on T2-weighted images, isointense to slightly hypointense on T1-weighted images, and shows reduced diffusion (hyperintensity on diffusion-weighted images and low apparent diffusion coefficient [ADC] values) without contrast enhancement during the acute period of the disease (1). Splenial lesions may extend into the callosal radiations, frontoparietal subcortical white matter and to the rest of the corpus callosum. Type I refers to isolated splenial involvement, while type II refers to additional involvement of remainder of the corpus callosum or supratentorial white matter $(4,5)$.

The pathogenesis of MERS is not known. Some bacterial/viral agents (rotavirus, adenovirus, influenza A and B, dengue virus, mumps virus, Mycoplasma pneumoniae, Streptococcus pneumoniae) and hyponatremia are the most commonly reported associations with MERS $(2,6-11)$. Prognosis is usually favorable with complete resolution of clinical and imaging findings within several months. 
Although many of the reports of MERS have been from East Asia, especially from Japan, there are case reports and case series of MERS from European countries including United Kingdom (8), Switzerland (12), Spain (13), Turkey $(10,14)$ and also from Australia (15) and Malaysia (9). Herein, we present clinical and MRI findings of 8 Turkish children with MERS and emphasize the clinical and MRI findings of this syndrome.

\section{Methods}

Institutional review board approval was obtained, and the need for informed consent was waived for this retrospective study.

Children who were admitted to the hospital from September 2015 to June 2017 with mild encepalitis/encephalopathy with complete recovery associated with MRI findings of a reversible lesion with transiently reduced diffusion isolated within the splenium of corpus callosum (SCC), within the SCC and subcortical frontoparietal, or involving the entire corpus callosum and subcortical frontoparietal white matter were reviewed from both radiology and pediatric neurology databases. Eight children with the diagnosis of MERS ( 5 boys and 3 girls; median age, 5.9 years; age range, 8 months to 14.1 years) were included in the study. We reviewed the patients' data retro-

Main points

- Mild encephalitis/encephalopathy with a reversible splenial lesion (MERS) is an acute encephalopathy state with characteristic diagnostic MRI findings.

- Presenting neurologic symptoms of MERS generally include disturbance of consciousness, delirious behavior, seizures, drowsiness, headache, monoparesis, abnormal speech, visual hallucinations, and ataxia. Transient blindness may also be seen.

- On MRI, MERS is almost always associated with a transient splenial lesion that is slightly hyperintense on T2-weighted images, isointense to slightly hypointense on T1-weighted images. Lesions show reduced diffusion without contrast enhancement. They may be limited to splenium (type l, ovoid or band shaped) or extend into callosal radiations, frontoparietal subcortical white matter, to the rest of the corpus callosum, and even cerebellum (type 2).

- Clinical and radiologic outcome is generally favorable but patients, especially those with type II lesions on MRI, may develop neurologic sequelae and lesions may persist on MRI for months even if their size diminishes independently of neurologic sequelae.

Table. MRI findings of patients presenting with MERS

\begin{tabular}{|c|c|c|c|c|}
\hline Patient & $\begin{array}{l}\text { Time to first MRI } \\
\text { after the onset } \\
\text { of symptoms }\end{array}$ & $\begin{array}{l}\text { Lesion type } \\
\text { (location, shape) }\end{array}$ & $\begin{array}{l}\text { Time to follow-up } \\
\text { MRI after the onset } \\
\text { of the symptoms }\end{array}$ & $\begin{array}{l}\text { Follow-up } \\
\text { MRI findings }\end{array}$ \\
\hline 1 & 1 day & I (SCC, ovoid) & 37 days & $\mathrm{CR}$ \\
\hline 2 & 2 days & $\begin{array}{l}\text { II (SCC and } \\
\text { posterior } \\
\text { periventricular } \\
\text { white matter) }\end{array}$ & 42 days & $\mathrm{CR}$ \\
\hline 3 & 1 day & I (SCC, ovoid) & 10 days & CR \\
\hline 4 & 1 day & I (SCC, ovoid) & 117 days & CR \\
\hline 5 & 1 day & I (SCC, ovoid) & 21 days & CR \\
\hline 6 & 1 day & I (SCC, band) & None & CR \\
\hline 7 & 1 day & I (SCC, ovoid) & 21 days & CR \\
\hline 8 & 1 day & I (SCC, ovoid) & 30 days & CR \\
\hline
\end{tabular}

spectively including demographic characteristics, prodromal and initial neurologic symptoms, neurologic examination, MRI and electroencephalography (EEG) findings, laboratory findings, treatment, and prognosis.

All MRI examinations included T2-weighted imaging, fluid attenuated inversion recovery (FLAIR), T1-weighted imaging, contrast-enhanced T1-weighted imaging, diffusion-weighted imaging (DWI), and apparent diffusion coefficient (ADC) maps and were reviewed by a senior pediatric radiologist. MRI examinations were performed on Optima MR450w 1.5 Tesla GE MRI scanner. Six children who needed sedation were sedated with chloral hydrate. Dotarem ${ }^{\circledast}$ (gadoterate meglumine, Guerbet) was used as a gadolinium-based contrast agent in all children. Follow-up MRI examinations were performed in 7 patients after 10 to 110 days. EEG follow-up was performed on the third day of symptoms if there was an initial abnormality.

\section{Results}

All patients were previously healthy. Nonspecific prodromal symptoms were present in 7 , including nausea and vomiting $(n=6)$, diarrhea $(n=6)$, and fever $(n=3)$. Acute encephalopathy was present in all patients. Initial neurologic symptoms were seizures $(n=4)$, delirious behavior $(n=1)$, drowsiness $(n=1)$, ataxia $(n=1)$, transient blindness $(n=2)$, abnormal speech $(n=2)$, and head- ache $(n=1)$. Initial neurologic examination findings were normal in 6 patients; one patient was confused and had myoclonic jerks on left arm with nuchal rigidity and another had nuchal rigidity.

Serum inflammatory markers, white blood cell (WBC) counts and C-reactive protein (CRP) levels were elevated in 6 patients (WBC $>10^{\circ} / \mathrm{L}, \mathrm{CRP}>0.5 \mathrm{mg} / \mathrm{dL}$ ). Both WBC and CRP levels were raised in Patient 5 who had a urinary tract infection caused by $E$. coli. Seven patients had hyponatremia (<135 mmol/L).

Two patients had a suspected infective agent: one had positive urine culture for E. coli and one was positive for rotavirus, using rapid antigen-detection assay in the stool. Cerebrospinal fluid (CSF) examinations (available in 6 patients) revealed normal cell counts, protein and glucose levels except in 2 patients where glucose was slightly elevated. There was no growth in CSF bacterial cultures.

MRI was performed within 1-2 days of the onset of neurologic symptoms. Symmetrical ovoid ( $n=6)$ (Fig. 1) and bandshaped $(n=1)$ (Fig. 2) lesions at the splenium of the corpus callosum (type I) were seen in 7 patients. One patient (Patient 2) had symmetrical periatrial lesions in addition to a splenial lesion (type II) (Fig. 3). The margins of the lesions were sharply outlined. On T2-weighted imaging, the lesions were homogeneously hyperintense and displayed restricted diffusion. The lesions were isointense to mildly hypointense on 
T1-weighted imaging and did not show enhancement. Follow-up scans were performed 10-110 days after the initial exam-
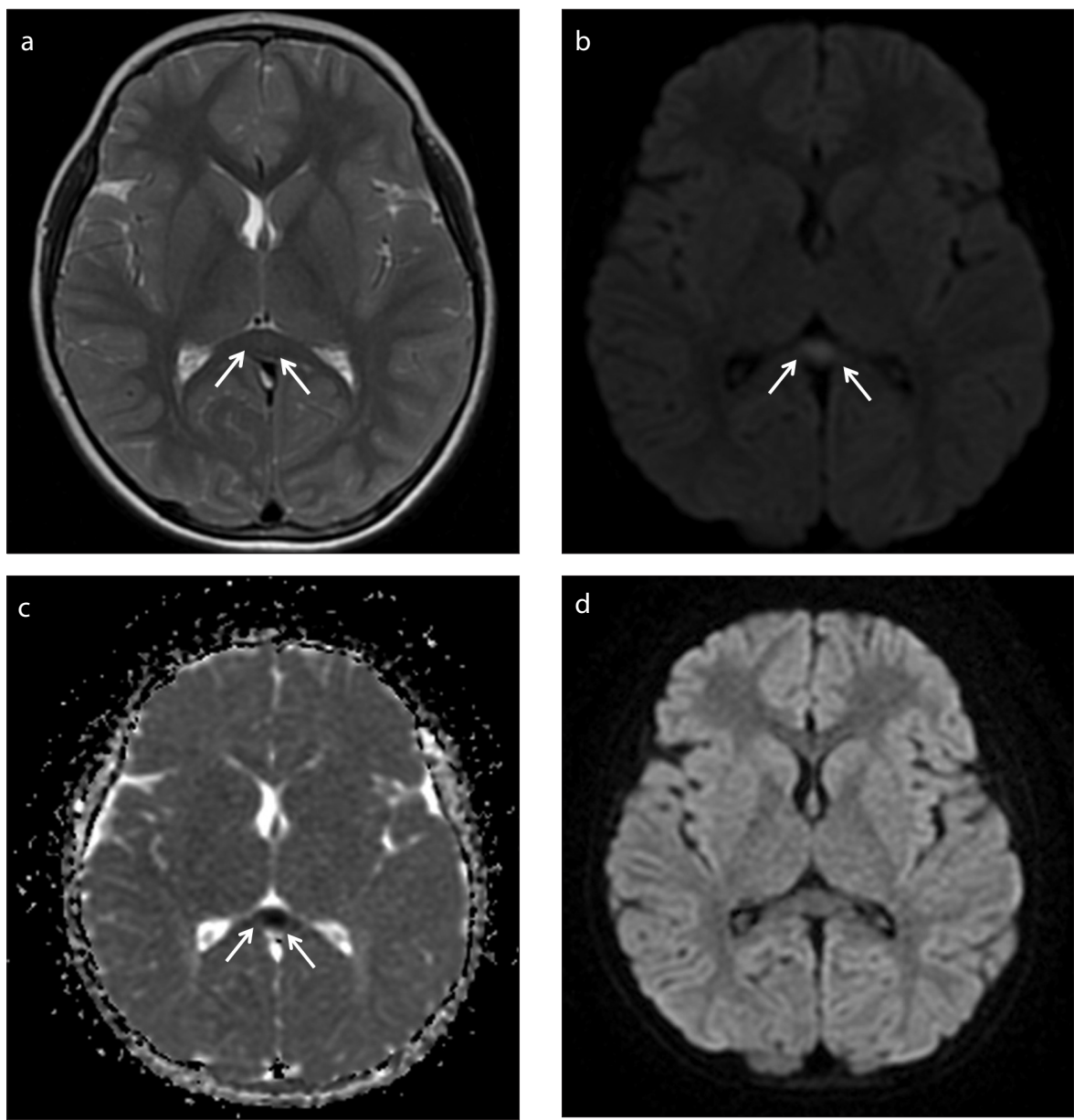

Figure 1. a-d. A 7-year-old male presented with delirium and speech abnormality after vomiting and diarrhea. His first brain MRI showed central ovoid lesion at splenium of corpus callosum (arrows) (type I). Lesion was slightly hyperintense on T2-weighted image (a), hyperintense on diffusion-weighted image (b) and hypointense on ADC map related to diffusion restriction (c). Follow-up MRI showed complete resolution of the lesion on diffusion-weighted image (d). initial EEG (obtained on day 1) was abnormal, showing global diffuse slow waves, which normalized on follow-up EEG obtained on day 3.

Two patients whose initial symptoms were transient blindness did not receive any treatment. Two patients were treated with anti-epileptic drugs. Three patients received antibiotic (ceftriaxone) and antiviral treatment (acyclovir) for 2 days until negative CSF culture and PCR results were shown. One patient who had a positive urine culture for E. coli, continued the treatment with ceftriaxone for 10 days. One patient who had sinusitis was treated with ampicillin/sulbactam for 10 days.

In all patients, neurologic symptoms resolved completely within 1-2 days from the onset of symptoms.

\section{Discussion}

MERS is an acute encephalopathy state with characteristic diagnostic MRI findings. Although a great majority of the cases were reported from East Asia, it can be seen worldwide. The data from our country is limited to a few case reports. In this report, we describe the largest case series of MERS reported from Turkey $(10,14)$.

Prodromal symptoms of children suffering from MERS most reported in the English literature are fever, vomiting, diarrhea, cough, abdominal pain, and headache $(2,4,5,7-12$, 14-18). In a study of 29 patients with MERS, nonspecific prodromal symptoms included fever $(n=22)$, vomiting $(n=14)$, diarrhea $(n=9)$, abdominal pain $(n=3)$, and cough $(n=4)$ (3). The prodromal symptoms of our patients were generally similar to those re-
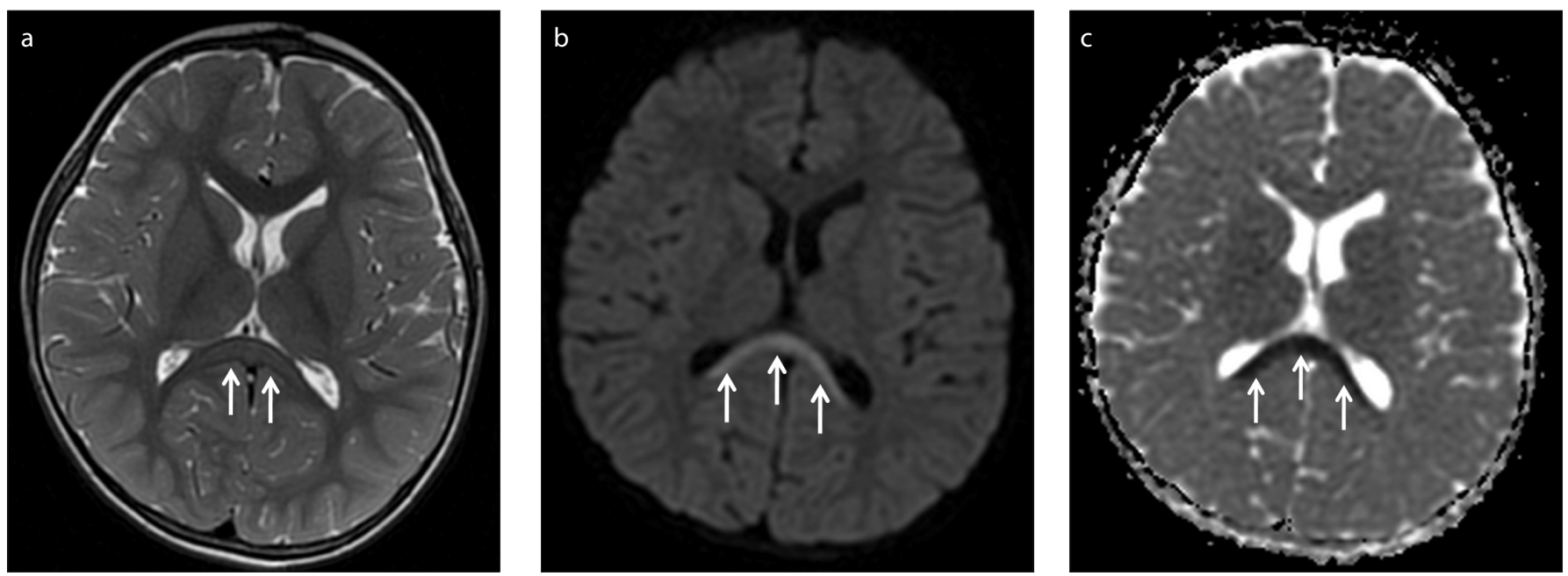

Figure 2. a-c. A three and a half years old male presented with seizure after vomiting and diarrhea. His first brain MRI showed band-shaped lesion at splenium of corpus callosum (arrows) (type I). Lesion was slightly hyperintense on T2-weighted image (a), hyperintense on diffusion-weighted image (b) and hypointense on ADC map related to diffusion restriction (c). Follow-up MRI was not performed. 

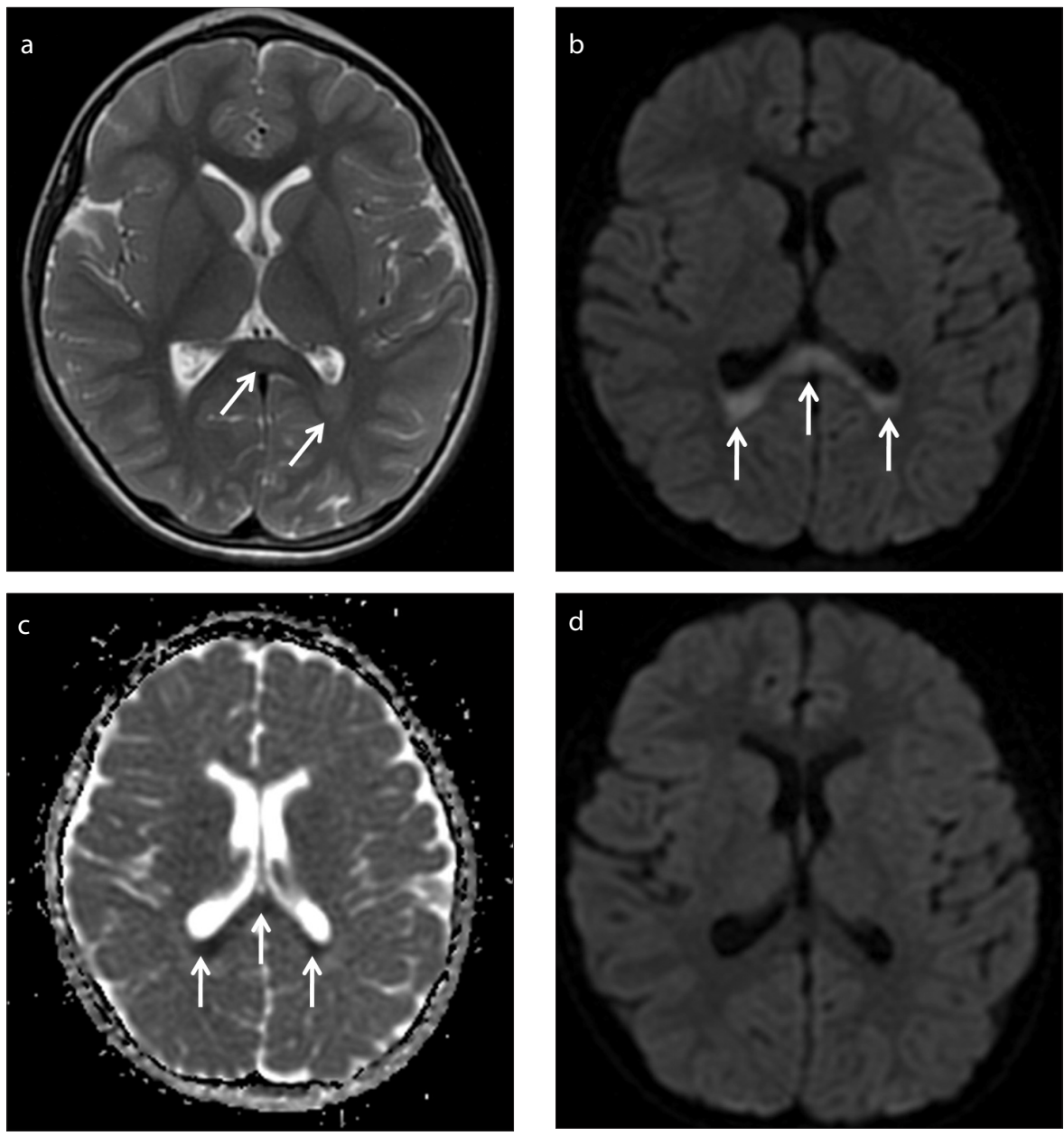

Figure 3. a-d. A 6-year-old male presented with speech abnormality and seizure. His first brain MRI showed symmetric posterior periventricular lesions in addition to splenial lesion (arrows) (type II). Lesion was slightly hyperintense on T2-weighted image (a), hyperintense on diffusion-weighted image (b) and hypointense on ADC map related to diffusion restriction (c). Follow-up MRI showed complete resolution of the lesion on diffusion-weighted image (d).

ported in the literature, but nausea and vomiting with diarrhea were more prominent (6 of 8 patients), while fever was less frequent (3 of 8 patients).

Presenting neurologic symptoms of MERS generally include disturbance of consciousness, delirious behavior, seizures, drowsiness, headache, monoparesis, abnormal speech, visual hallucinations, and ataxia $(2,4,5,7-12,14-18)$. Seizures were the most common presenting symptoms in our patients. Transient blindness was seen in 2 of 8 patients and lasted for minutes. In the literature transient blindness was reported as an initial symptom of MERS in a few children (3). Although the number of patients in our study was low, the rate of patients with transient blindness was higher than the number given in the literature (3).

The corpus callosum is the biggest fiber bundle, with projections into prefrontal, premotor, primary motor, and primary sen- sory areas. The disturbance in the collasal connections can cause disorder of motor control, spatial orientation, vision, hearing, and language-related behaviors (19). These may explain the neurologic symptoms of MERS, including blindness.

Lack of pleocytosis in the CSF supports the hypothesis that MERS is an infection-associated encephalopathy syndrome rather than an encephalitis. Pleocytosis in MERS has been reported inconsistently (2, 11). Ueda et al. (2) and Takanashi et al. (11) found pleocytosis in 33\% (3/9) and 100\% $(5 / 5)$ of their patients respectively. On the other hand, lower pleocytosis ratios similar to our results (i.e., $14.3 \%, 10 \%$, and $7.7 \%$ ) were reported by Ka et al. (15), Fang et al. (3) and Chen et al. (7), respectively.

Many MERS patients, without any attributed causative agent in CSF cultures, have been reported to have infection at other foci. In this regard, further possible explanation for transient cerebral edema is myelin-specific neurotoxin release causing inflammatory infiltrate by a pathogen. Both viral and bacterial non-CNS infections have been reported in MERS patients. MERS-related viruses include rotavirus, adenovirus, influenza A and B, Epstein-Barr virus, Dengue virus, mumps virus, herpes simplex virus, parainfluenza, parvovirus B-19 and cytomegalovirus; additionally, bacterial infections with Mycoplasma pneumoniae, Streptococcus pneumoniae, salmonella, and Campylobacter jejuni have also been reported in some MERS cases. (4, 7-11, 12, 15, $17,18)$. On the contrary, only two patients were shown to have infectious agents in our study (Patient 5: E. coli and Patient 8: rotavirus). Although we could not show the infectious agent, Patient 2 had sinusitis that was diagnosed based on symptoms and MRI findings showing air-fluid levels.

Hyponatremia is another possible etiologic factor in MERS. The rate of hyponatremic patients in our study (87.5\%) was similar to the rate reported by Takanashi et al. (6), where 25 of 30 patients with MERS had $\mathrm{Na}<136 \mathrm{mmol} / \mathrm{L}$. It is postulated that hypotonic hyponatremia may cause intramyelinic edema causing transient reduced diffusion seen on MRI (6). Diarrhea and vomiting may cause imbalances in $\mathrm{Na}$ levels, but in another case presented by Sato et al. (20), the effect of dehydration is also emphasized. They reported single photon emission computed tomography (SPECT) findings of a patient with MERS and demonstrated hypoperfusion of the bilateral cingulate gyri, thalamus, basal ganglia, brainstem, and cortex of the frontal lobes. According to this finding they defended the opinion that cerebral hypoperfusion leads to neurologic symptoms and MRI findings.

MRI findings we report are similar to those reported previously. The lesions typically demonstrate hyperintensity on T2-weighted imaging, isointensity to slight hypointensity on T1-weighted imaging, and show reduced diffusion (hyperintensity on DWI and low ADC values) without contrast enhancement. Seven of our patients had type I lesions, where only the splenium of the corpus callosum was symmetrically involved. Type II lesions may extend to the remainder of the corpus callosum, periventricular white matter, centrum semiovale, and involve even the cerebellum $(2,4,5,11$, 15). As observed in our patients, the lesions generally resolve completely on follow-up imaging $(2,3,11)$. However, type II lesions 
may persist for months even if their size diminishes $(2,15,16)$.

The most common EEG abnormality is global diffuse slow waves, as seen in one patient in our series $(5,8,11,12,16)$. Fang et al. (3) and Kashiwagi et al. (18) reported EEG abnormalities in $43 \%-48 \%$ of MERS cases.

All of our patients recovered completely without any clinical sequelae at discharge. Clinical improvement was seen within 1-2 days. Radiologic improvement was shown at 10 days to 4 months. However, the timing of full normalization on MRI is not known since frequent follow-up imaging was not performed. In the literature, patients with MERS are generally reported to recover completely both clinically and on imaging $(8-11,3,12,16,17)$. However, some patients with type II lesions can develop neurologic sequelae. Ueda et al. (2) reported one patient with type II lesions who developed neurologic sequelae, which resolved 2 months after onset. The patient had slight disability of fine motor incoordination of the left hand. Ka et al. (15) reported two patients with type II lesions who developed neurologic sequelae. The patients' residual ataxia improved in 1 to 4 weeks after discharge.

Although transient splenic lesions triggered by infectious agents are often described as MERS, the distinction between MERS and other transient splenial lesions is unclear $(5,6)$. Transient splenial lesions, except for infectious and metabolic causes, have also been reported in various conditions including epilepsy, antiepileptic drug therapy or withdrawal, malnutrition, vitamin B12 deficiency, high altitude edema, alcohol poisoning, eclampsia, carbon monoxide poisoning, and migraine (13, 21-23).

Acute disseminated encephalomyelitis (ADEM) should be considered in the differential diagnosis of any patient with acute encephalopathy. Although clinical presentations of MERS and ADEM are similar, MERS can be differentiated from ADEM on the basis of MRI findings. ADEM causes asymmetrical white matter lesions that do not demonstrate diffusion restriction and persist for months on MRI beyond clinical resolution of the disease (15).
The main limitations of our study are its retrospective nature and the small sample size. Five patients had vomiting and diarrhea but we could not demonstrate a possible pathogen. However, compared with the results of reported cases and case series in the literature, our study revealed similar results.

In conclusion, MERS is an acute encephalopathy state with a favorable outcome. Children may present with a wide spectrum of neurologic symptoms including temporary blindness. Radiologists should be aware of its characteristic MRI findings which normalize in days to months.

\section{Conflict of interest disclosure}

The authors declared no conflicts of interest.

\section{References}

1. Tada H, Takanashi J, Barkovich AJ, et al. Clinically mild encephalitis/encephalopathy with a reversible splenial lesion. Neurology 2004; 63:1854-1858. [CrossRef]

2. Ueda N, Minami S, Akimoto M. Mycoplasma pneumoniae-associated mild encephalitis/encephalopathy with a reversible splenial lesion: report of two pediatric cases and a comprehensive literature review. BMC Infect Dis 2016; 16:671. [CrossRef]

3. Fang $Q$, Chen $L$, Chen $Q$, Lin Z, Yang F. Clinically mild encephalitis/encephalopathy with a reversible splenial lesion of corpus callosum in Chinese children. Brain Dev 2017; 39:321-326. [CrossRef]

4. Takanashi J, Barkovich AJ, Shiihara T, et al. Widening spectrum of a reversible splenial lesion with transiently reduced diffusion. AJNR Am J Neuroradiol 2006; 27:836-838.

5. Takanashi J, Imamura A, Hayakawa F, Terada H. Differences in the time course of splenial and white matter lesions in clinically mild encephalitis/ encephalopathy with a reversible splenial lesion (MERS). J Neurol Sci 2010; 292:24-27. [CrossRef]

6. Takanashi J, Tada H, Maeda M, Suzuki M, Terada $\mathrm{H}$, Barkovich AJ. Encephalopathy with a reversible splenial lesion is associated with hyponatremia. Brain Dev 2009; 31:217-220. [CrossRef]

7. Chen WX, Liu HS, Yang SD, et al. Reversible splenial lesion syndrome in children: Retrospective study and summary of case series. Brain Dev 2016; 38:915-927. [CrossRef]

8. Karampatsas K, Spyridou C, Morrison IR, Tong $\mathrm{CY}$, Prendergast AJ. Rotavirus-associated mild encephalopathy with a reversible splenial lesion (MERS)-case report and review of the literature. BMC Infect Dis 2015; 15:446. [CrossRef]

9. Fong $C Y$, Khine $M M$, Peter $A B$, Lim WK, Rozalli FI, Rahmat K. Mild encephalitis/encephalopathy with reversible splenial lesion (MERS) due to dengue virus. J Clin Neurosci 2017; 36:73-75. [CrossRef]
10. Avcu G, Kilinc MA, Eraslan C, Karapinar B, Vardar F. Mild encephalitis/encephalopathy with reversible splenial lesion (MERS) associated with Streptococcus pneumoniae Bacteraemia. J Infect Public Health 2017; 10:479-482. [CrossRef]

11. Takanashi J, Shiihara T, Hasegawa T, et al. Clinically mild encephalitis with a reversible splenial lesion (MERS) after mumps vaccination. J Neurol Sci 2015; 349:226-228. [CrossRef]

12. Abenhaim Halpern L, Agyeman $P$, Steinlin $M$, ElKoussy M, Grunt S. Mild encephalopathy with splenial lesion and parainfluenza virus infection. Pediatr Neurol 2013; 48:252-254. [CrossRef]

13. Garcia-Monco JC, Cortina IE, Ferreira E, Martínez A, Ruiz L, Cabrera A, Beldarrain MG. Reversible splenial lesion syndrome (RESLES): what's in a name? J Neuroimaging 2011; $21:$ e114. [CrossRef]

14. Bulakbasi N, Kocaoglu M, Tayfun C, Ucoz T. Transient splenial lesion of the corpus callosum in clinically mild influenza-associated encephalitis/encephalopathy. AJNR Am J Neuroradiol 2006; 27:1983-1986.

15. Ka A, Britton P, Troedson C, et al. Mild encephalopathy with reversible splenial lesion: an important differential of encephalitis. Eur J Paediatr Neurol 2015; 19:377-382. [CrossRef]

16. Hashimoto $Y$, Takanashi J, Kaiho K, et al. A splenial lesion with transiently reduced diffusion in clinically mild encephalitis is not always reversible: A case report. Brain Dev 2009; 31:710-712. [CrossRef]

17. Suzuki H, Kusaka T, Okada H. Clinically mild encephalitis/encephalopathy with a reversible splenial lesion caused by human parvovirus b19 infection: a case of two brothers with hereditary spherocytosis. Pediatr Neurol 2014; 51:470-472. [CrossRef]

18. Kashiwagi $M$, Tanabe $T$, Shimakawa $S$, et al. Clinico-radiological spectrum of reversible splenial lesions in children. Brain Dev 2014; 36:330-336. [CrossRef]

19. Jea A, Vachhrajani $S$, Widjaja E, et al. Corpus callosotomy in children and the disconnection syndromes: a review. Childs Nerv Syst 2008; 24:685-692. [CrossRef]

20. Sato T, Ushiroda Y, Oyama T, et al. Kawasaki disease-associated MERS: pathological insights from SPECT findings. Brain Dev 2012; 34:605608. [CrossRef]

21. Fuseya Y, Komatsu K, Matsumoto S. Transient splenial lesion following abrupt withdrawal of carbamazepine. Intern Med 2017; 56:989-990. [CrossRef]

22. Göçmen $\mathrm{R}$, Ünal $E$. Transient splenial lesion in a case with carbon monoxide poisoning: A clue supporting the excitotoxicity hypothesis? Eur J Paediatr Neurol 2015; 19:716-718. [CrossRef]

23. Ünver O, Kutlubay B, Besci T, Ekinci G, Baltacıoğlu F, Türkdoğan D. Transient splenial lesion of the corpus callosum related to migraine with aura in a pediatric patient. Acta Medica (Hradec Kralove) 2016; 59:64-66. [CrossRef] 